\title{
Motor cortex neurovascular coupling: inputs from ultra-high-frequency ultrasound imaging in humans
}

\author{
Fabien Almairac, MD, PhD, ${ }^{1}$ Denys Fontaine, MD, PhD, ${ }^{1,2}$ Thomas Demarcy, $\mathrm{PhD},{ }^{3}$ \\ Hervé Delingette, $\mathrm{PhD},{ }^{3}$ Stéphanie Beuil, MD, ${ }^{4}$ and Charles Raffaelli, MD ${ }^{4}$
}

Departments of ${ }^{1}$ Neurosurgery and ${ }^{4} \mathrm{Ultra}$-Sound Imaging, CHU de Nice, Université Cote d'Azur, Nice; ${ }^{2} \mathrm{FHU}$ INOVPAIN, CHU de Nice, Université Cote d'Azur, Nice; and ${ }^{3}$ Asclepios Research Team, INRIA Sophia Antipolis-Mediterranée, France

\begin{abstract}
OBJECTIVE Neurovascular coupling reflects the link between neural activity and changes in cerebral blood flow. Despite many technical advances in functional exploration of the brain, including functional MRI, there are only a few reports of direct evidence of neurovascular coupling in humans. The authors aimed to explore, for the first time in humans, the local cerebral blood flow of the primary motor cortex using ultra-high-frequency ultrasound (UHF-US) Doppler imaging to detect low blood flow velocity $(1 \mathrm{~mm} / \mathrm{sec})$.
\end{abstract}

METHODS Four consecutive patients underwent awake craniotomy for glioma resection using cortical direct electrostimulation for brain mapping. The primary motor cortical area eliciting flexion of the contralateral forearm was identified. UHF-US color Doppler imaging of this cortical area was acquired at rest, during repeated spontaneous forearm flexion, and immediately after the movement's termination. In each condition, the surface areas of the detectable vessels were measured after extraction of non-zero-velocity colored pixels and summed.

RESULTS During movement, local cerebral blood flow increased significantly by $14.4 \%$ (range 5\%-30\%) compared with baseline. Immediately after the termination of movements, the local hyperemia decreased significantly by $8.6 \%$ (range $1.9 \%-15.7 \%)$.

CONCLUSIONS To the authors' knowledge, this study is the first to provide a real-time demonstration of the neurovascular coupling in the human cortex by ultrasound imaging. They assume that UHF-US may be used to gather original and advanced data on brain functioning, which could be used to help in the identification of functional cortical areas during brain surgery.

Clinical trial registration no.: NCT03179176 (clinicaltrials.gov)

https://thejns.org/doi/abs/10.3171/2018.5.JNS18754

KEYWORDS cerebral blood flow; neurovascular coupling; imaging methodology; ultrasound; motor cortex; clinical neurophysiology; diagnostic technique

$\mathrm{T}$ To ensure appropriate blood supply through the brain, the cerebral blood vessels, neurons, and astrocyte glial cells must interact according to complex mechanisms. This interaction, named "neurovascular coupling" (NVC), describes the link between neuronal activity and cerebral blood flow (CBF) changes. ${ }^{25}$

Several methods have been developed to assess the NVC in humans by exploring either cerebral tissue oxygenation using functional MRI, near-infrared spectroscopy, or positron emission tomography, or CBF changes using transcranial Doppler (TCD) ultrasonography., ${ }^{9,2}$ TCD ultrasonography is the ultrasound (US) imaging technique mostly used in the clinic to explore CBF because of its high temporal resolution and noninvasive nature. For example, sensorimotor or cognitive stimulatory effects on cerebral blood velocity have been studied using low-frequency ( $\leq$ 2-MHz) transducers. ${ }^{4}$ In healthy individuals, this response leads to an average $10 \%-20 \%$ increase in CBF in the posterior cerebral artery during visual stimulation, ${ }^{1}$ and a 5\%-8\% increase in the middle cerebral artery during a cognitive task. ${ }^{22}$ However, despite good spatiotemporal resolution, TCD US imaging is limited to the exploration of large cerebral vessels and is not able to detect subtle changes of low blood flow in cortical vessels that may occur during a task.

Recently, very high frame rate US imaging $(>10,000$ frames per second) was shown to enable high-resolution and high-sensitivity power Doppler imaging, ,29 allowing

ABBREVIATIONS BOLD = blood oxygen level-dependent; $C B F=$ cerebral blood flow; DES = direct electrostimulation; fUS = functional US; mSA = median surface area; NVC = neurovascular coupling; TCD = transcranial Doppler; UHF-US = ultra-high-frequency US; US = ultrasound.

SUBMITTED March 19, 2018. ACCEPTED May 31, 2018.

INCLUDE WHEN CITING Published online November 9, 2018; DOI: 10.3171/2018.5.JNS18754. 
the detection of low blood velocities $(<4 \mathrm{~mm} / \mathrm{sec})$ that occur in arterioles and venules of the rat brain. The concept of functional ultrasound (fUS) has been proposed regarding the ability to visualize whole-brain microvasculature dynamics in response to cerebral activation with a high spatiotemporal resolution. In a rat model, after craniotomy, fUS has been used to image sensory cortex and thalamic responses after whisker stimulation and propagation of epileptiform seizures. ${ }^{15}$ It was sensitive enough to detect odor-evoked $\mathrm{CBF}$ changes in the rodent olfactory bulb. ${ }^{18}$ However, the skull is a limit for cerebral fUS due to the poor penetration of US waves through the bone. ${ }^{23}$

The recent development and introduction of latest-generation ultra-high-frequency ultrasound (UHF-US) linear transducers (up to $70 \mathrm{MHz}$ ) in clinical use ${ }^{3}$ may extend the US applications to explore brain functioning. Here, we provide the first study that aims to explore NVC of the human motor cortex with new UHF-US Doppler imaging (Vevo MD, Visualsonics), allowing a resolution of $50 \mu \mathrm{M}$ up to $12.5 \mathrm{~mm}$ deep, and the detection by Doppler of the cortical microvasculature flow $<1 \mathrm{~mm} / \mathrm{sec}$ in patients undergoing awake craniotomy for brain tumor resection. We also aimed to investigate the feasibility of and potential interest in UHF-US as a tool to explore brain functions during neurosurgical procedures.

\section{Methods}

\section{Participants and Surgical Procedure}

All patients included in the study underwent exploration using UHF-US during awake craniotomy procedures performed for brain tumor resection requiring brain mapping by direct electrostimulation (DES). In this study, approved by a national ethics committee and registered (clinical trial registration no.: NCT03179176, clinicaltrials.gov), we did not modify the usual surgical and brain mapping protocols.

DES was used to identify crucial neural structures for brain functioning during tumor resection to preserve them and to optimize the extent of resection, ${ }^{5}$ with consideration of individual functional boundaries. Language and sensorimotor skills were checked continuously during the awake period while the neurosurgeon applied DES at the cortical and subcortical levels (bipolar electrode, biphasic current, $60 \mathrm{~Hz}, 1.5-$ to $4-\mathrm{mA}$ intensity). ${ }^{28} \mathrm{DES}$ of the motor cortex and underlying corticospinal tract provoked a transient, involuntary movement of the contralateral stimulated body, with respect to somatotopy and a high spatial accuracy $(5 \mathrm{~mm}){ }^{24}$

The patient's head was immobilized in a 3-pin head frame. After opening the skull, neurosedation was stopped and the patient was awakened. Brain mapping was performed before tumor resection to identify eloquent cortical regions to be preserved. The primary motor area was localized by DES. The primary motor area with DES-induced flexion of the contralateral forearm was identified precisely (Fig. 1). Patients whose tumor invaded the primary motor area were excluded.

\section{UHF-US Imaging}

UHF-US images were acquired using Vevo MD (FUJIFILM Visualsonics), a recent US imaging device that obtains UHF-US images with color Doppler acquisitions. This device is CE marked for human use. We used the $46-\mathrm{MHz}$ linear-array transducer $(30-\mathrm{MHz}$ center frequency), allowing axial and lateral resolution of $50 \mu \mathrm{M}$ and $70 \mu \mathrm{M}$, respectively, and detection of the microvasculature flow $<1 \mathrm{~mm} / \mathrm{sec}$ (manufacturer's specifications). The Doppler characteristics were as follows: transmitter frequency, $21 \mathrm{MHz}$; frame acquisition rate, 9 per second; and exploration depth, $12.5 \mathrm{~mm}$. The Doppler mode was set up at $1.8 \mathrm{~cm} / \mathrm{sec}$; beam angle, $0^{\circ}$; wall filter, low; and persistence, median.

\section{UHF-US Image Acquisition}

UHF-US images were acquired when the patient was awakened and fully compliant. The transducer was placed within a sterile sheath in contact with the cortex, exactly on the motor area previously identified to induce forearm flexion (Fig. 1). The transducer was fixed to a custom articulated arm to avoid movements during image acquisition. Image acquisition was performed by 2 radiologists (C.R. and S.B.) who are experts in US use.

First, morphological acquisitions were performed without Doppler (10-second duration) to visualize the sulci, gyri, and the underlying white matter on a surface of 1 $\mathrm{cm}^{2}$. Then, Doppler acquisitions were carried out in 3 distinct conditions. In the first condition, the patient was at rest ("rest1" condition; 15 to 55 seconds). Next, the patient was asked to reproduce exactly the same forearm movements ("movement" condition) as those elicited by DES, repeatedly (26 seconds to 1 minute). Finally, the patient was asked to stop the movement and to stay at rest ("rest2" condition) until the end of the Doppler acquisition time (20 seconds to 1 minute). The total acquisition time was short (about 5 minutes per patient) and did not interfere with the continuation of the surgery or the patient's comfort.

\section{Imaging Treatment and Analysis}

Imaging sequences acquired during the surgery were analyzed a posteriori. Image processing consisted in converting the RGB (red, green, blue) DICOM image to its chroma component, $\mathrm{C}=\operatorname{maximum}(\mathrm{R}, \mathrm{G}, \mathrm{B})-\operatorname{minimum}(\mathrm{R}, \mathrm{G}, \mathrm{B})$, and thresholding it at $12.5 \%(\mathrm{C}>0.125)$. This allowed us to extract pixels from the color-coded Doppler overlay on top of a grayscale B-mode US image. The surface area of all vessels detectable in the volume explored by UHF-US was evaluated by summing all pixels with a nonzero velocity and taking into account the "PhysicalDeltaX" and "PhysicalDeltaY" DICOM tags to be converted into square millimeters (Fig. 1). To smooth the oscillatory short-term variations of the native curve, related to hemodynamic variations induced by heart pulsations, the median surface area (mSA) value was graphically represented over a 5-second period (-2.5 seconds; +2.5 seconds).

\section{Statistical Analysis}

ANOVA was conducted on surface area values of each condition (rest1, movement, rest2). As these values were not normally distributed (inequality of variance), a nonparametric Kruskal-Wallis test was used. For multiple pairwise comparisons of the surface area values for each 

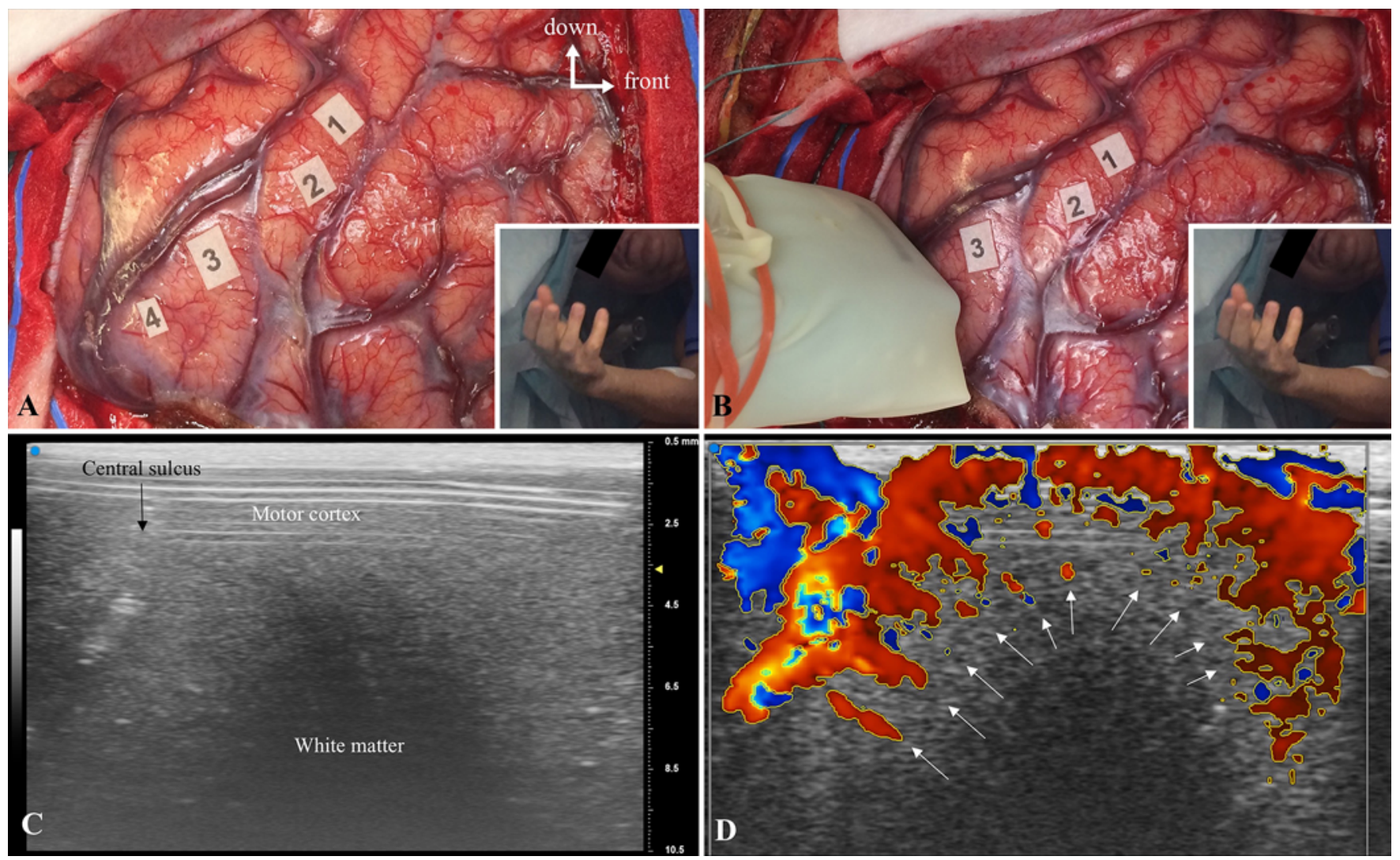

FIG. 1. Study methods, UHF-US image acquisition, and processing. A: Intraoperative photograph of the left motor area and functional mapping using DES. Tags 1-4 are located in the precentral gyrus. DES applied on the tag 4 cortical site induced flexion of the contralateral forearm (inset). B: Intraoperative photograph showing the UHF-US Doppler probe within the sterile sheath positioned on cortical site 4, during spontaneous continuous flexion of the contralateral forearm (inset). C: Morphological UHF-US image acquired over the motor area (tag 4) showing the central sulcus (black arrow), the precentral gyrus, and the underlying white matter. D: Image processing after UHF-US color Doppler imaging, acquired over the motor area tag 4, showing pial superficial and sulcal vessels and their penetrating branches (white arrows). All colored pixels with a nonzero velocity (delineated in yellow) were extracted and summed to be converted into square millimeters. Figure is available in color online only.

condition, Bonferroni correction was applied. The statistical threshold was set at $p<0.05$. All statistical analyses were performed with XLSTAT software version 2017.4 (Addinsoft).

\section{Results}

Four consecutive patients (26-41 years old, mean 31.5 years) with a low-grade glioma located close to eloquent areas were included and underwent surgery (Fig. 2).

Doppler sequences recorded at rest and during movements allowed the visualization of the macro- and microvasculature of the cortex to a maximum depth of $12.5 \mathrm{~mm}$. The arteries could not be distinguished from the veins. Results are displayed in Table 1 and Fig. 3.

At rest1, the mSA values recorded by Doppler were between $0.261 \mathrm{~mm}^{2}$ and $0.452 \mathrm{~mm}^{2}$. During movements, mSA values were between $0.328 \mathrm{~mm}^{2}$ and $0.475 \mathrm{~mm}^{2}$. The local CBF, estimated by mSA, increased significantly compared with the rest1 condition (mean mSA increase $14.4 \%$ [range 5\%-30\%], $\mathrm{p}<0.001$ ). At rest 2 , the mean mSA decreased significantly by $8.6 \%$ (range $1.9 \%-15.7 \%$, $\mathrm{p}<0.001)$ compared with the movement condition.

\section{Discussion}

In our experimental conditions, local hyperemia of the human primary motor cortex during the voluntary movements was observed in real time by color Doppler UHFUS imaging. To our knowledge, these findings are the first direct demonstration of the NVC in the human cortex by using US imaging.

The UHF-US technology, with linear-array transducers cadenced at more than $30 \mathrm{MHz}$ (up to $70 \mathrm{MHz}$ ), allows a temporal and spatial resolution much higher than conventional US systems available in clinical practice. ${ }^{19,29}$ Ultra-high-resolution US (with a 46- to 70-MHz lineararray transducer) was recently used to explore the anatomy of nails and their microvasculature. ${ }^{3}$ It seems to offer several advantages over existing noninvasive imaging modalities, especially in terms of easy, advanced, and noninvasive visualization of various superficial targets within the first 1-cm depth below the skin surface. In neuroscience, although US is widely used in clinics for blood flow imaging, its role in real time has been limited, until now, to the exploration of large vessels. As UHF-US can explore the microvasculature at the cortical level, it may be used to 
A
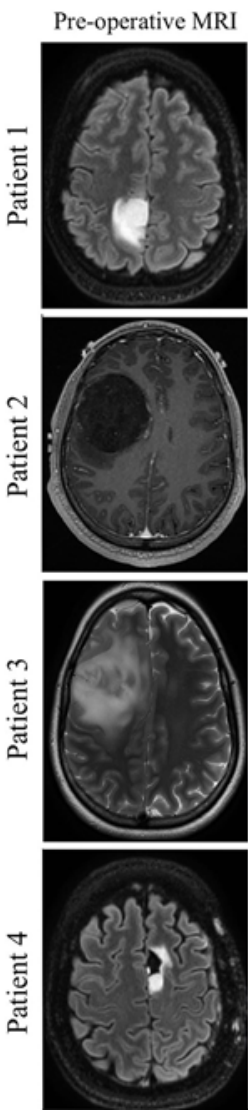

B
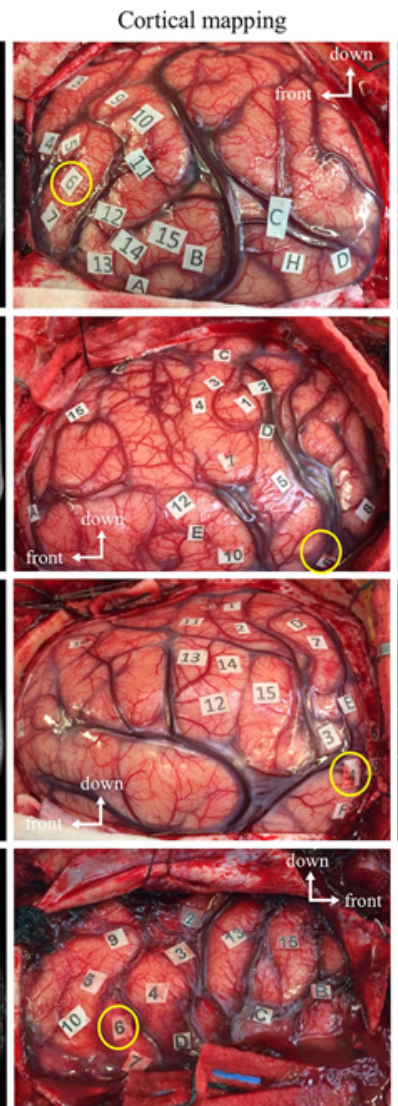

C
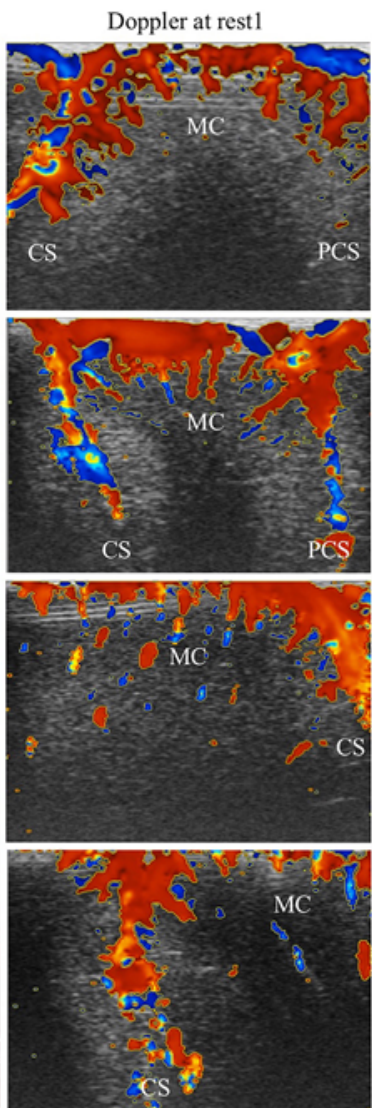

D

Doppler during the movements
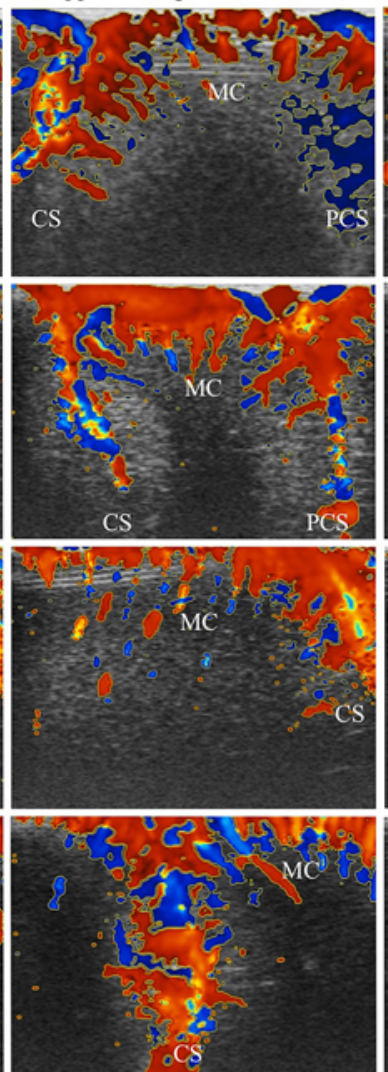

E

Doppler at rest2
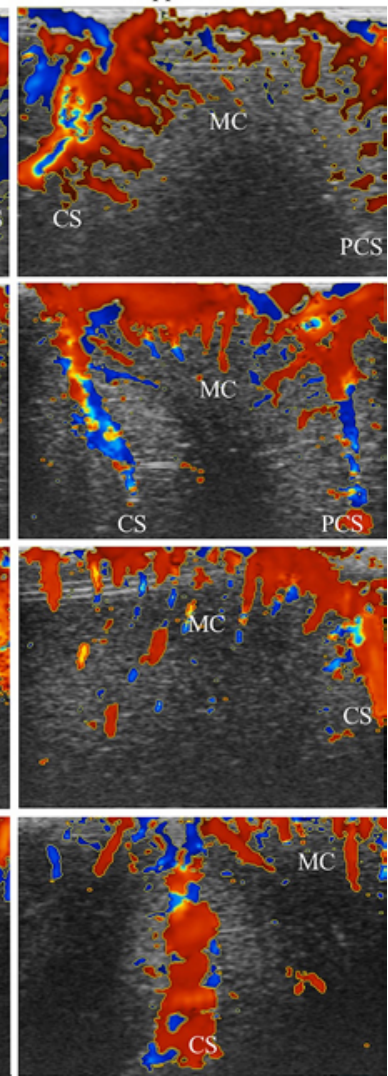

FIG. 2. Motor cortex CBF changes during movements in UHF-US in 4 patients who underwent awake craniotomy for tumor resection. A: Preoperative MR images showing the location of the brain tumors. B: Intraoperative photographs showing cortical mapping using DES. Yellow circles indicate motor cortical sites where DES induced forearm flexion, which were further explored using UHF-US. C: UHF-US color Doppler imaging at rest 1 before movements. D: UHF-US color Doppler imaging during repeated forearm flexion. Local CBF in the motor area increased during movements compared with rest1 and rest2 conditions. E: UHF-US color Doppler imaging at rest2 after termination of movements. $C S=$ central sulcus; $M C=$ motor cortex; PCS = precentral sulcus. Figure is available in color online only.

TABLE 1. Surface area of the cortical vessels measured on UHF-US Doppler images in 4 patients during rest and movement

\begin{tabular}{|c|c|c|c|c|c|c|c|c|}
\hline \multirow[b]{2}{*}{ Patient No. } & \multirow[b]{2}{*}{ Value Type } & \multicolumn{3}{|c|}{ Surface Area, mm² } & \multirow[b]{2}{*}{$\% \Delta \mathrm{M}-\mathrm{R} 1$} & \multirow[b]{2}{*}{$p$ Value } & \multirow[b]{2}{*}{$\% \Delta \mathrm{M}-\mathrm{R} 2$} & \multirow[b]{2}{*}{ p Value } \\
\hline & & Rest1 & Movement & Rest2 & & & & \\
\hline \multirow{3}{*}{1} & Median & 0.323 & 0.368 & 0.310 & \multirow{3}{*}{+12.26} & \multirow{3}{*}{$<0.001$} & \multirow{3}{*}{-15.71} & \multirow{3}{*}{$<0.001$} \\
\hline & Max & 0.391 & 0.435 & 0.435 & & & & \\
\hline & Min & 0.257 & 0.260 & 0.254 & & & & \\
\hline \multirow{3}{*}{2} & Median & 0.452 & 0.475 & 0.466 & \multirow{3}{*}{+4.95} & \multirow{3}{*}{$<0.001$} & \multirow{3}{*}{-2.08} & \multirow{3}{*}{$<0.001$} \\
\hline & Max & 0.490 & 0.50 & 0.484 & & & & \\
\hline & Min & 0.340 & 0.327 & 0.330 & & & & \\
\hline \multirow{3}{*}{3} & Median & 0.294 & 0.328 & 0.322 & \multirow{3}{*}{+10.34} & \multirow{3}{*}{$<0.001$} & \multirow{3}{*}{-1.89} & \multirow{3}{*}{$<0.001$} \\
\hline & Max & 0.308 & 0.353 & 0.339 & & & & \\
\hline & Min & 0.265 & 0.281 & 0.304 & & & & \\
\hline \multirow{3}{*}{4} & Median & 0.261 & 0.373 & 0.318 & \multirow{3}{*}{+30.03} & \multirow{3}{*}{$<0.001$} & \multirow{3}{*}{-14.79} & \multirow{3}{*}{$<0.001$} \\
\hline & Max & 0.335 & 0.428 & 0.398 & & & & \\
\hline & Min & 0.238 & 0.327 & 0.270 & & & & \\
\hline
\end{tabular}

Min = minimum; $\max =$ maximum; Rest $1=$ resting time before the movements period; Rest2 = resting time after the termination of movements; $\Delta \mathrm{M}-\mathrm{R} 1=$ difference in surface area values between movement and rest1; $\Delta \mathrm{M}-\mathrm{R} 2$ = difference in surface area values between movement and rest2.

The nonparametric ANOVA test was used to compare the surface area values for each condition, with multiple comparisons after Bonferroni correction. 

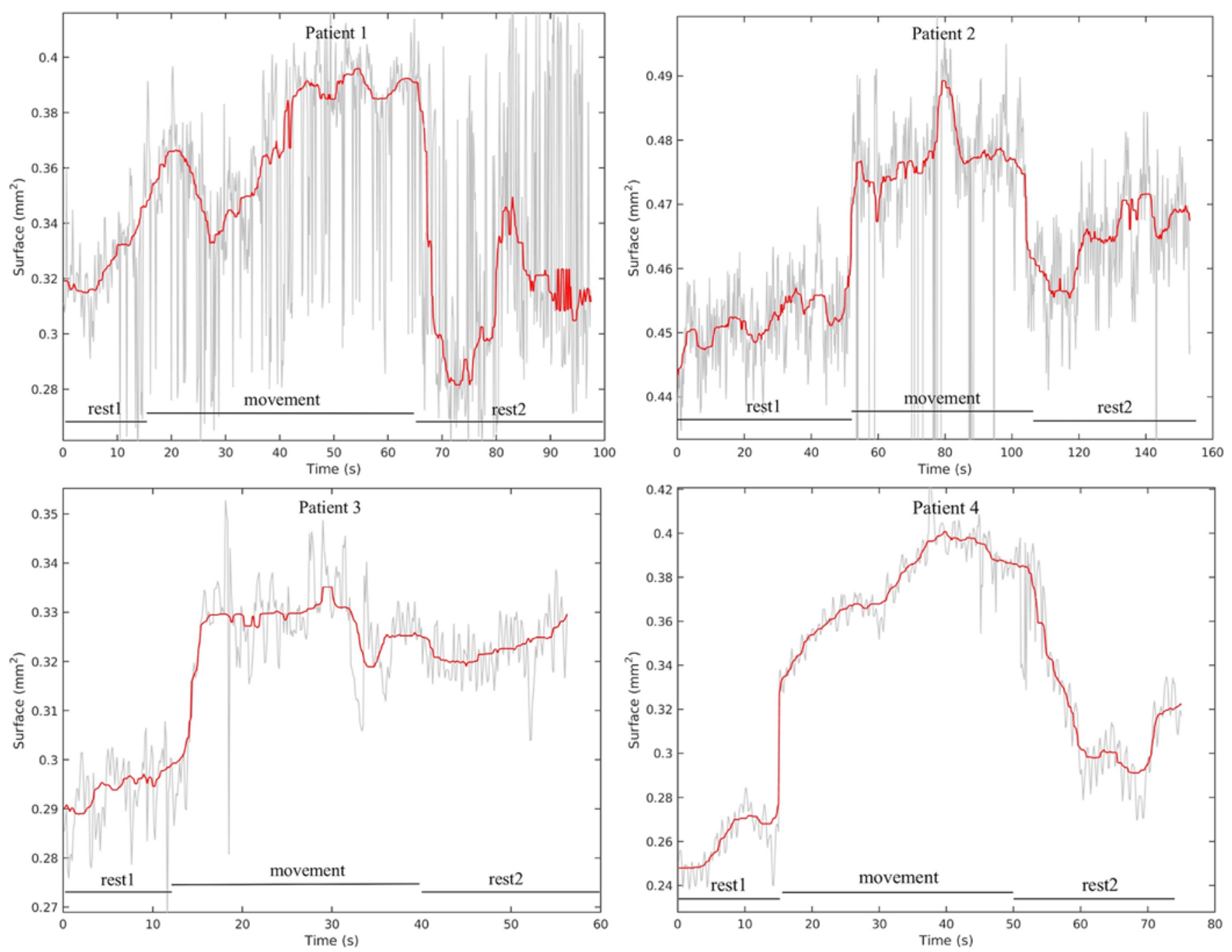

FIG. 3. Changes in the surface area of the vessels, measured on UHF-US Doppler images, during rest and movements, in 4 patients who underwent awake craniotomy. The "rest1," "movement," and "rest2" annotations and their underlying segments represent the time duration of each experimental condition. Red curve: median value of vessels' surface area over a 5-second period $(-2.5$ seconds; +2.5 seconds). Gray curve: absolute value of vessels' surface area. Figure is available in color online only.

gather original and advanced data on brain functioning. In our study, we identified changes in the microvascularization, resulting in $\mathrm{CBF}$ changes, within a portion of the motor cortex associated with its functional activation. These findings obtained with UHF-US technology might extend the use of intraoperative US neuroimaging to real-time exploration of brain functioning.

As previously shown in animals and humans, we observed a reversible increase of local CBF during functional activation of the cortex, followed by a decrease. Although the amplitude of these CBF changes varied across the patients (i.e., variations of mSA increased by $5 \%$ to $30 \%$, and decreased by $1.9 \%$ to $15.7 \%$ ), the mean mSA increase (14.4\%) was similar to those observed using ultrafast Doppler $(20 \%)^{10}$ and in former US studies (between 5\% and 20\%). ${ }^{1,21}$ The observed interpatient variations could be explained by several factors, such as different positioning of the US probe on the cortex, anatomical variations of the precentral gyrus, different orientation of the vessels with regard to the transducer, individual factors influencing he- modynamics ${ }^{6}$ and hemodynamic response to neural activation, ${ }^{16}$ and, most of all, the proportion of neural tissue actually activated within the volume of cortex explored by the UHF-US window. ${ }^{20}$

The aim of our pilot feasibility study was to assess the ability of the UHF-US scanner to detect and quantify hemodynamic changes in the cortex and therefore it had several limitations.

UHF-US with Doppler imaging explores flow velocity within blood vessels and displays them as colored pixels, with a color scale reflecting the velocity. We recorded and quantified the total surface area of pixels obtained with color Doppler imaging and inferred that it was a reflection of the vessels' surface area, varying with the diameter and the number of vessels. Changes in vessel diameter can be extrapolated directly from the surface area and could be objectified by direct visualization. Intuitively and subjectively, the number of vessels visible on the Doppler images increased during movement, but we were not able to quantify reliably this number and its changes. First, only ves- 
sels with a flow velocity superior to the detection threshold were visible. Second, due to a related effect, quantification of vessels by pixel contouring was not reliable; many contoured vessels that were isolated from each other in the rest condition joined during the movements when their diameter increased, leading to an artificial and contradictory decrease in the number of measured pixels. In addition, unlike some studies in animals,$^{15}$ our study failed to provide quantitative values of other hemodynamic parameters, such as blood velocity or blood volume. Indeed, due to the threshold effect on the color Doppler mode (set at $1.8 \mathrm{~cm} / \mathrm{sec}$ ), the aliasing observed when the flow was less than $-1.8 \mathrm{~cm} / \mathrm{sec}$ or greater than $+1.8 \mathrm{~cm} / \mathrm{sec}$ may have prevented the extrapolation of values required for the reliable quantitative estimation of the CBF or velocity, since the color code (red for positive values, blue for negative ones) was disrupted below or above $1.8 \mathrm{~cm} / \mathrm{sec}$. In addition, using 2D technology the angle of exploration of the many vessels, which can vary due to several previously cited factors, could not be extrapolated. These 2 elements prevented the extrapolation of quantitative values of the blood flow.

Although the motor task-induced $\mathrm{CBF}$ changes were easily identified after image processing (Fig. 3), their detection was more subtle on the screen of the device, as seen in Fig. 2. In order to help the neurosurgeon detect taskinduced CBF variations in real time, a simple algorithm for detecting variations of the vessel surface area (e.g., the one used in the present study) could be developed in the future and implemented in the device software. A necessary validation phase on a set of patients in a dedicated study would thus be addressed.

Finally, we did not explore precisely the dynamics of CBF changes, as the onset of neuronal activation was not monitored precisely by electrocorticography, ${ }^{27}$ intracortical electrophysiology (i.e., local field potentials), ${ }^{14}$ or 2-photon microscopy, ${ }^{16}$ but considered these changes as being the moment when the patient was asked to perform the arm movement. It should also be noted that the detailed mechanisms of NVC and its exact linkage with neuronal firing are still debated and may not be a perfect surrogate of neuronal activity. ${ }^{8,26}$ The addition of electrophysiological recordings of the cortex to UHF-US exploration is necessary in future studies to clarify the relationship between $\mathrm{CBF}$ visualized on US imaging and neuronal activity.

Despite these limitations, we demonstrated cortical CBF changes related to cortical activation for the first time with UHF-US ( $>30 \mathrm{MHz}$ ) in patients. By providing very high spatial resolution with a sufficient frame rate (wider than a cardiac cycle), UHF-US seems to be a powerful device for studying the cortex microvasculature in intraoperative conditions and discriminating thin functional areas. Such NVC has been explored in rodents and in humans using the ultrafast Doppler technology with similar findings. In a rat model, fUS showed an increase in CBF in the sensory cortex and thalamus after whisker stimulation $^{15}$ and CBF increase in the motor area after inferior limb movement..$^{19}$ Using a method similar to ours (i.e., US cortical acquisition in patients after craniotomy), Imbault et al. demonstrated the ability of ultrafast Doppler tech- nology to identify, map, and differentiate regions of brain activation in patients during task-evoked cortical response within the depth of a sulcus. ${ }^{10}$ However, unlike Doppler UHF-US images, ultrafast Doppler images require image processing after ultrasonography so that $\mathrm{CBF}$ changes can be displayed as activation maps; as a consequence, the cortical vasculature changes cannot be detected in real time during the surgery, limiting the use of ultrafast Doppler in operating rooms. Moreover, compared with UHF-US imaging, the ultrafast Doppler technology using a 6-MHz probe allows deeper exploration of the brain, but at the expense of the detection quality of the surface microvasculature.

Other imaging modalities in humans are available to detect NVC. Regional CBF changes in $\mathrm{H}_{2} \mathrm{O}$ PET scanning have been used to explore various functions, ${ }^{11}$ showing an increase in cortical and subcortical regional CBF related to task activation, similar to our observations with UHFUS imaging. More recently, functional MRI uses the blood oxygen level-dependent (BOLD) effect, reflecting the local oxygen consumption rather than CBF. However, in some studies, the accuracy of task-based functional MRI compared with DES is reported to be limited, particularly for language-related functions. ${ }^{7}$ Furthermore, the relationship between BOLD response parameters and neuronal activity is still a matter of intense research..$^{12,13,17}$ These issues regarding functional imaging methods strengthen the need to correlate UHF-US findings (i.e., task-induced $\mathrm{CBF}$ changes) with cortical neuronal activity, not only for motor-related functions but also for other brain functions, such as sensation, language, cognition, and vision, in a translational perspective. Also, it could be interesting to compare the task-induced BOLD signal with task-induced CBF changes controlled by DES responses in a dedicated study.

Compared with these functional imaging modalities, UHF-US imaging has the advantage of better spatial and temporal resolution, but requires craniotomy and explores a limited brain superficial area.

UHF-US could be specially adapted to intraoperative functional exploration. Indeed, beyond the research applications, through the development and the validation of this technology, it could be an interesting complementary method to the benchmark of awake surgery with DES. It could be used to identify the hidden parts of functional cortices that cannot be explored by DES due to accessibility issues, such as the depth of the sulci and basal and medial portions of the cortex.

\section{Conclusions}

In our exploratory study, the high temporal and spatial accuracies of UHF-US imaging combined with the high functional specificity of DES (identification of functional cortical motor area responsible for a defined movement) allowed us to demonstrate the NVC in the human motor cortex. To our knowledge, these findings represent the first real-time demonstration of NVC in the human cortex by US imaging. We assume that UHF-US imaging may be used to gather original and advanced data on brain functioning and could be used to help with the identification of functional cortical areas during brain surgery. 


\section{Acknowledgments}

We thank the University Hospital of Nice for having promoted this study.

\section{References}

1. Aaslid R: Visually evoked dynamic blood flow response of the human cerebral circulation. Stroke 18:771-775, 1987

2. Bercoff J, Montaldo G, Loupas T, Savery D, Mézière F, Fink $\mathrm{M}$, et al: Ultrafast compound Doppler imaging: providing full blood flow characterization. IEEE Trans Ultrason Ferroelectr Freq Control 58:134-147, 2011

3. Berritto D, Iacobellis F, Rossi C, Reginelli A, Cappabianca S, Grassi R: Ultra high-frequency ultrasound: New capabilities for nail anatomy exploration. J Dermatol 44:43-46, 2017

4. D'Andrea A, Conte M, Scarafile R, Riegler L, Cocchia R, Pezzullo E, et al: Transcranial Doppler ultrasound: physical principles and principal applications in neurocritical care unit. J Cardiovasc Echogr 26:28-41, 2016

5. De Witt Hamer PC, Robles SG, Zwinderman AH, Duffau H, Berger MS: Impact of intraoperative stimulation brain mapping on glioma surgery outcome: a meta-analysis. J Clin Oncol 30:2559-2565, 2012

6. Duarte JV, Pereira JMS, Quendera B, Raimundo M, Moreno C, Gomes L, et al: Early disrupted neurovascular coupling and changed event level hemodynamic response function in type 2 diabetes: an fMRI study. J Cereb Blood Flow Metab 35:1671-1680, 2015

7. Giussani C, Roux FE, Ojemann J, Sganzerla EP, Pirillo D, Papagno C: Is preoperative functional magnetic resonance imaging reliable for language areas mapping in brain tumor surgery? Review of language functional magnetic resonance imaging and direct cortical stimulation correlation studies. Neurosurgery 66:113-120, 2010

8. Hillman EMC: Coupling mechanism and significance of the BOLD signal: a status report. Annu Rev Neurosci 37:161181,2014

9. Huneau C, Benali H, Chabriat H: Investigating human neurovascular coupling using functional neuroimaging: a critical review of dynamic models. Front Neurosci 9:467, 2015

10. Imbault M, Chauvet D, Gennisson JL, Capelle L, Tanter M: Intraoperative functional ultrasound imaging of human brain activity. Sci $\operatorname{Rep~7:7304,~} 2017$

11. Kameyama M, Murakami K, Jinzaki M: Comparison of $\left[{ }^{15} \mathrm{O}\right]$ $\mathrm{H}_{2} \mathrm{O}$ positron emission tomography and functional magnetic resonance imaging in activation studies. World $\mathbf{J}$ Nucl Med 15:3-6, 2016

12. Lippert MT, Steudel T, Ohl F, Logothetis NK, Kayser C: Coupling of neural activity and fMRI-BOLD in the motion area MT. Magn Reson Imaging 28:1087-1094, 2010

13. Logothetis NK: What we can do and what we cannot do with fMRI. Nature 453:869-878, 2008

14. Logothetis NK, Pauls J, Augath M, Trinath T, Oeltermann A: Neurophysiological investigation of the basis of the fMRI signal. Nature 412:150-157, 2001

15. Macé E, Montaldo G, Cohen I, Baulac M, Fink M, Tanter M: Functional ultrasound imaging of the brain. Nat Methods 8:662-664, 2011

16. O'Herron P, Chhatbar PY, Levy M, Shen Z, Schramm AE, Lu Z, et al: Neural correlates of single-vessel haemodynamic responses in vivo. Nature 534:378-382, 2016

17. Ojemann GA, Ojemann J, Ramsey NF: Relation between functional magnetic resonance imaging (fMRI) and single neuron, local field potential (LFP) and electrocorticography (ECoG) activity in human cortex. Front Hum Neurosci 7:34, 2013

18. Osmanski BF, Martin C, Montaldo G, Lanièce P, Pain F, Tanter M, et al: Functional ultrasound imaging reveals different odor-evoked patterns of vascular activity in the main olfactory bulb and the anterior piriform cortex. Neuroimage 95:176-184, 2014

19. Osmanski BF, Pezet S, Ricobaraza A, Lenkei Z, Tanter M: Functional ultrasound imaging of intrinsic connectivity in the living rat brain with high spatiotemporal resolution. Nat Commun 5:5023, 2014

20. Penfield W, Jasper H: Epilepsy and the Functional Anatomy of the Human Brain. London: J. \& A. Churchill Ltd., 1954

21. Phillips AA, Chan FH, Zheng MMZ, Krassioukov AV, Ainslie PN: Neurovascular coupling in humans: physiology, methodological advances and clinical implications. J Cereb Blood Flow Metab 36:647-664, 2016

22. Phillips AA, Warburton DER, Ainslie PN, Krassioukov AV: Regional neurovascular coupling and cognitive performance in those with low blood pressure secondary to high-level spinal cord injury: improved by alpha-1 agonist midodrine hydrochloride. J Cereb Blood Flow Metab 34:794-801, 2014

23. Pinton G, Aubry JF, Bossy E, Muller M, Pernot M, Tanter M: Attenuation, scattering, and absorption of ultrasound in the skull bone. Med Phys 39:299-307, 2012

24. Rasmussen T, Penfield W: Further studies of the sensory and motor cerebral cortex of man. Fed Proc 6:452-460, 1947

25. Roy CS, Sherrington CS: On the regulation of the bloodsupply of the brain. J Physiol 11:85-108, 158-7-158-17, 1890

26. Sheth SA, Nemoto M, Guiou M, Walker M, Pouratian N, Toga AW: Linear and nonlinear relationships between neuronal activity, oxygen metabolism, and hemodynamic responses. Neuron 42:347-355, 2004

27. Siero JCW, Hermes D, Hoogduin H, Luijten PR, Petridou N, Ramsey NF: BOLD consistently matches electrophysiology in human sensorimotor cortex at increasing movement rates: a combined 7T fMRI and ECoG study on neurovascular coupling. J Cereb Blood Flow Metab 33:1448-1456, 2013

28. Szelényi A, Bello L, Duffau H, Fava E, Feigl GC, Galanda $\mathrm{M}$, et al: Intraoperative electrical stimulation in awake craniotomy: methodological aspects of current practice. Neurosurg Focus 28(2):E7, 2010

29. Tanter M, Fink M: Ultrafast imaging in biomedical ultrasound. IEEE Trans Ultrason Ferroelectr Freq Control 61:102-119, 2014

\section{Disclosures}

Dr. Demarcy: employee of Oticon Medical.

\section{Author Contributions}

Conception and design: Almairac, Fontaine, Raffaelli. Acquisition of data: Almairac, Fontaine, Beuil, Raffaelli. Analysis and interpretation of data: Almairac, Fontaine, Demarcy, Beuil, Raffaelli. Drafting the article: Almairac, Fontaine. Critically revising the article: Almairac, Fontaine, Demarcy, Raffaelli. Reviewed submitted version of manuscript: Almairac, Fontaine, Raffaelli. Approved the final version of the manuscript on behalf of all authors: Almairac. Statistical analysis: Almairac, Demarcy. Administrative/technical/material support: Almairac, Fontaine, Delingette, Beuil, Raffaelli. Study supervision: Almairac, Fontaine.

\section{Supplemental Information \\ Previous Presentations}

Portions of this work were presented in oral form at the European Association of Neurosurgical Societies (EANS) 2017 Annual Meeting, Venice, Italy, October 3, 2017.

\section{Correspondence}

Fabien Almairac: Hôpital Pasteur 2, Nice, France. almairac.f@ chu-nice.fr. 